\title{
MODEL FOR PREDICTING BATHYMETRIC AND GRAIN SIZE CHANGES BASED ON BAGNOLD'S CONCEPT AND EQUILIBRIUM SLOPE CORRESPONDING TO GRAIN SIZE COMPOSITION
}

\author{
Yasuhito Noshi' ${ }^{1}$, Takaaki Uda ${ }^{2}$, Masumi Serizawa ${ }^{3}$, Takayuki Kumada ${ }^{4}$ and Akio Kobayashi ${ }^{5}$
}

\begin{abstract}
A model for predicting bathymetric and grain size changes considering the equilibrium slopes corresponding to each grain size and its composition was incorporated into the BG model proposed by Serizawa et al. (2007). The model was applied to predict the beach changes at Kemigawa Beach. The cross-shore sorting of sand, in which the grain size gradually decreases with increasing depth, and the formation of a gradually changing longitudinal slope were accurately reproduced. The model was also applied to predict the selective accumulation of fine sand in a navigation channel after dredging and its impact on the nearby coast.
\end{abstract}

Keywords: prediction model; Bagnold's concept; equilibrium slope; navigation channel; bathymetric change; grain size change

\section{INTRODUCTION}

In general, grain size sorting occurs under wave action; coarser materials accumulate near the shoreline, forming a steep slope, whereas fine sand tends to be deposited further offshore with a gentle slope. On the basis of these phenomena, Uda et al. (2004) developed a contour-line-change model for predicting changes in the longitudinal profile by considering the equilibrium slope corresponding to each grain size. Kumada et al. (2006) expanded the model, assuming that the longitudinal profile can be expressed as a combination of several equilibrium slopes that are given by each grain size population. The applicability of their model to practical problems is high, and Kumada et al. (2007) applied the model when designing the most effective shape of an artificial headland to prevent sand transport to Oharai Port. In their method, however, the equilibrium slope does not change, even if the content of each grain size in the population slightly changes, since each equilibrium slope is assumed to be invariant, and therefore, it is difficult to predict spatial changes in the longitudinal profile associated with changes in the composition of each grain size. In fact, the longitudinal slope gradually becomes gentle with increasing content of fine sand, for example, in the wave-shelter zone, but these changes in the local slope in and around the wave-shelter zone due to this mechanism cannot be predicted by their model. Noshi et al. (2009) improved the model using the contour-line-change model and taking the equilibrium slope corresponding to each grain size and its composition into account. Although the applicability of this contour-line-change model to practical problems is high, the handling of complicated boundary conditions around various structures is more difficult compared with the BG model proposed by Serizawa et al. (2007), in which the fundamental equations are solved in a mesh. In this study, Noshi et al.'s modification of the contour-line-change model is incorporated into the BG model, which has an advantage of being able to predict topographic changes near the structures with complicated shape. To verify the applicability of the new model, it is applied to the bathymetric changes of Kemigawa Beach and is used to investigate the mechanism of the selective accumulation of fine sand into the navigation channel of a port after dredging as well as its impact on the nearby coast.

\section{MODEL}

Fluid motion near the sea bottom under wave action is oscillatory, and therefore, the back and forth motion of the bottom sediment is generated as a result of the fluid motion. Inman and Bagnold (1963) assumed that the sand transport under wave action is given by the sum of two components: shoreward sand transport caused by incoming waves and seaward sand transport caused by outgoing waves, and they derived a sand transport equation, which explicitly includes the seabed slope, based on the energy conservation law. By applying the concept of the equilibrium slope introduced by Inman and Bagnold (1963) and the energetics approach of Bagnold (1963), Serizawa et al. (2006) proposed Eq. (1) for the

\footnotetext{
${ }^{1}$ Dr. Eng., ICOMNET, Ltd. 2-7-4-627 Aomi, Koto-ku, Tokyo 135-0064, Japan

2 Dr. Eng., Executive Director, Public Works Research Center, 1-6-4 Taito, Taito, Tokyo 110-0016, Japan

${ }_{3}^{3}$ Coastal Engineering Laboratory Co., Ltd., 1-22-301 Wakaba, Shinjuku, Tokyo 160-0011, Japan

${ }^{4}$ Dr. Eng., Public Works Research Center, 1-6-4 Taito, Taito, Tokyo 110-0016, Japan

${ }^{5}$ Dr. Eng., Department of Oceanic Architecture \& Engineering, College of Science \& Technology, Nihon University,

7-24-1 Narashinodai, Funabashi, Chiba 274-8501, Japan
} 
transport flux of sand composed of a single grain size using only the wave conditions at the breaking point.

$$
\begin{gathered}
\vec{q}=\frac{G}{\tan \beta_{\mathrm{c}}}\left[\tan \beta_{\mathrm{c}} \overrightarrow{e_{w}}-\overrightarrow{\nabla Z}\right] \\
G=C_{0} K_{1} \varepsilon(Z)\left(E C_{g}\right)_{b} \cos ^{2} \alpha_{b} \tan \beta_{\mathrm{c}}
\end{gathered}
$$

Here, $\beta_{\mathrm{c}}$ is the equilibrium slope angle, $\overrightarrow{\nabla z}=(\partial z / \partial x, \partial z / \partial y)$ is the gradient vector of seabed elevation, $\overrightarrow{e_{w}}$ is the unit vector of wave direction, $K_{1}$ is the coefficient of longshore sand transport, $\left(E C_{g}\right)_{b}$ is the energy flux at the breaking point, $\alpha_{b}$ is the breaker angle and $C_{0}$ is a coefficient used to transform the immersed weight of sand into a volumetric expression. $\varepsilon(Z)$ is the depth distribution of the intensity of sediment transport and is given by the cubic equation proposed by Uda et al. (1996).

Serizawa et al. (2007) expanded Eq. (1) so as to be applicable to the case of sand with mixed grain size referring to Uda et al. (2004), to develop a model for predicting changes in beach topography and grain size. Introducing the concept of a mixing layer proposed by Hirano (1971), the content $\mu^{(k)}$ of each grain size $d^{(k)}(k=1,2, \ldots, N)$ in the exchange layer is added to the variables to be solved. Sand transport equation for each grain size is given by Eq. (3) by multiplying Eq. (1) by the content of each grain size to evaluate its contribution to wave energy dissipation while introducing the equilibrium slope of each grain size. Furthermore, the longshore sand transport coefficient for each grain size (Eq. (5)) is assumed to be inversely proportional to the square root of the grain size referring to Kamphuis et al. (1986). The beach changes are determined by the sum of the components of the beach changes for each grain size, which are calculated by the continuity equation for each grain size (Eq. (6)). The content is calculated by solving the continuity equation in the exchange layer (Eq. (8)).

$$
\begin{gathered}
\vec{q}^{(k)}=\frac{G^{(k)}}{\tan \beta_{\mathrm{c}}{ }^{(k)}}\left(\tan \beta_{\mathrm{c}}{ }^{(k)} \overrightarrow{e_{w}}-\overrightarrow{\nabla Z}\right)(k=1,2, \ldots, N) \\
G^{(k)}=\mu^{(k)} \cdot C_{0} K_{1}^{(k)} \varepsilon(Z)\left(E C_{g}\right)_{b} \cos ^{2} \alpha_{b} \tan \bar{\beta} \quad(k=1,2, \ldots, N) \\
K_{1}^{(k)}=\frac{A_{1}}{\sqrt{d^{(k)}}} \quad(k=1,2, \ldots, N) \\
\frac{\partial z^{(k)}}{\partial t}=-\nabla \cdot \vec{q}(k) \quad(k=1,2, \ldots, N) \\
\frac{\partial \mu^{(k)}}{\partial t}=\left\{\begin{array}{l}
\frac{\partial Z}{\partial t}=\sum_{k=1}^{N} \frac{\partial z^{(k)}}{\partial t} \\
\frac{1}{B_{h}}\left(\frac{\partial z^{(k)}}{\partial t}-\mu^{(k)} \frac{\partial Z}{\partial t}\right) \quad \frac{\partial Z}{\partial t} \geq 0 \\
\left.\frac{\partial z_{h}^{(k)}}{\partial t}-\mu_{B}^{(k)} \frac{\partial Z}{\partial t}\right) \quad \frac{\partial Z}{\partial t}<0
\end{array} \quad(k=1,2, \ldots, N)\right.
\end{gathered}
$$

Here $N$ is the number of grain sizes, $B_{h}$ is the thickness of the exchange layer, $\mu^{(k)}: k=1,2, \ldots, N$ is the content of each grain size in the exchange layer, $\tan \beta_{c}^{(k)}$ is the equilibrium slope of each grain size, $K_{1}{ }^{(k)}$ is the coefficient of longshore sand transport corresponding to each grain size, $\tan \bar{\beta}: Z=-h_{C}-h_{R}$ is the mean slope of the initial cross section between $Z=-h_{c}$ and $h_{R}$, and $A_{1}$ is a constant depending on the condition of each specific coast. In addition, the grain size $d^{(k)}$ in Eq. (5) has a unit of mm. 
On a coast, not only the longshore sorting of the grain size of sand but also the cross-shore sorting of grain size can be simultaneously observed. Coarser material is deposited in the zone with a higher elevation on the foreshore due to the cross-shore sorting by waves, whereas fine sand tends to be deposited further offshore with increasing depth, and as a result, the parallel contours at the initial condition deform, forming a seabed slope that gradually becomes flatter from the erosion zone to the accretion zone.

Noshi et al. (2006) obtained the empirical relationship given by Eq. (9) to determine $\tan \beta_{c}$ using the content $\mu^{(k)}$ of each grain size and the equilibrium slope $\tan \beta_{c}^{(k)}$ corresponding to each grain size.

$$
\begin{gathered}
\tan \beta_{c}=\tan \beta_{\mathrm{c}}{ }^{(k)} \cdot r+\tan \overline{\beta_{\mathrm{c}}} \cdot(1-r) \\
\ln \left(\tan \bar{\beta}_{c}\right)=\sum_{k=1}^{N} \mu^{(i, k)} \ln \left(\tan \beta_{c}^{(k)}\right) \\
\ln \left(\tan \beta_{c}^{(k)}\right)=\ln a+b \ln \left(d^{(k)}\right)
\end{gathered}
$$

Then, Noshi et al. (2009) proposed the cross-shore sand transport equation for the contour-linechange model (Eq. (12)), using this method to calculate the local longitudinal slope corresponding to the composition of each grain size.

$$
\begin{aligned}
& q_{z}^{(k)}=\mu^{(k)} \cdot \varepsilon_{z}(z) \cdot \gamma \cdot K_{1}^{(k)} \cdot\left(E C_{g}\right)_{b} \cos ^{2} \alpha_{b s} \sin \bar{\beta} . \\
& \quad\left\{r \cdot\left(\cot \beta / \cot \bar{\beta}_{c}-1\right)+(1-r) \cdot\left(\cot \beta / \cot \beta_{c}^{(k)}-1\right)\right\} ; \\
& \quad k=1,2, \ldots, N \quad 0 \leq r \leq 1
\end{aligned}
$$

Here, $q_{z}^{(k)} ; k=1,2, \ldots, N$ is the cross-shore sand transport of each grain size and $\varepsilon(Z)$ is the depth distribution of the intensity of sand transport, $\tan \beta$ is the local seabed slope. The total cross-shore sand transport is given by the weighted mean of these cross-shore sand transports with weight $r$. In this study, $\mathrm{r}$ is set to 0.5 , assuming the same contribution of each term.

In this study, the sand transport equations for each grain size proposed by Serizawa et al. (Eqs. (3)(5)) were expanded on the basis of Noshi et al.'s concept. In formulating the sand transport equation, the coefficients of cross-shore and longshore sand transport were separately included in the basic equations similar to that in Uda et al. (2010).

$$
\begin{gathered}
q_{x}^{(k)}=\frac{G_{x}{ }^{(k)}}{\tan \beta_{\mathrm{c}}}\left(\tan \beta_{\mathrm{c}} \cos \theta_{w}-\frac{\partial Z}{\partial x}\right) \quad(k=1,2, \ldots, N) \\
q_{y}^{(k)}=\frac{G_{y}{ }^{(k)}}{\tan \beta_{\mathrm{c}}}\left(\tan \beta_{\mathrm{c}} \sin \theta_{w}-\frac{\partial Z}{\partial y}\right) \quad(k=1,2, \ldots, N) \\
G_{x}^{(k)}=K_{x}^{(k)} G^{(k)}, \quad G_{y}{ }^{(k)}=K_{y}{ }^{(k)} G^{(k)} \quad(k=1,2, \ldots, N) \\
G^{(k)}=\mu^{(k)} \cdot C_{0} \varepsilon(Z)\left(E C_{g}\right)_{b} \cos ^{2} \alpha_{b} \tan \bar{\beta} \quad(k=1,2, \ldots, N) \\
K_{y}{ }^{(k)}=\frac{A_{1}}{\sqrt{d^{(k)}}} \quad(k=1,2, \ldots, N) \\
\tan \beta_{c}=\tan \beta_{\mathrm{c}}{ }^{(k)} \cdot r+\tan \overline{\beta_{\mathrm{c}}} \cdot(1-r) \quad(k=1,2, \ldots, N)
\end{gathered}
$$

Here, $q_{x}$ is the cross-shore component of sediment transport flux (positive for shoreward), $q_{y}$ is the longshore component of sediment transport flux, $\theta_{w}$ is the angle between the wave direction and the $x$ axis, $K_{x}^{(k)}$ and $K_{y}{ }^{(k)}$ are the coefficients of cross-shore and longshore sand transport. The conservation of sand volume corresponding to each grain size and the content of each grain size in the exchange layer 
are the same as those in Serizawa et al. (2007). When offshore breakwaters are constructed, a waveshelter zone is formed behind these structures. In such cases, the wave diffraction coefficient and wave direction in the vicinity of the wave-shelter zone are calculated using the angular spreading method for irregular waves (Sakai et al., 2006) and then, the wave height is reduced by multiplying the breaker height without the offshore breakwaters by the wave diffraction coefficient. Furthermore, as the distribution of wave direction, the direction of diffracted waves is used. The additional longshore sand transport due to the longshore change in breaker height was evaluated by the method given by Ozasa and Brampton (1980).

\section{TOPOGRAPHIC CHANGES AT KEMIGAWA BEACH}

To verify the effectiveness of the proposed model, it was applied to predict the bathymetric changes of Kemigawa Beach facing Tokyo Bay, as shown in Fig. 1. Figure 2 shows the bathymetry of this beach measured in 2004. The straight, impermeable groins on both sides of the beach were extended by $230 \mathrm{~m}$ in 1979, and a 1,300-m-wide pocket beach with parallel contours was produced by beach nourishment. Then, a curved part was added to the groins in 1993, forming a large wave-shelter zone behind them. A Y-shaped groin was also constructed in the central part of the beach in 2002. As a result, the shoreline advanced in the wave-shelter zones formed behind the curved and $\mathrm{Y}$-shaped groins. This causes the deformation of the initial contours as shown in Fig. 2.

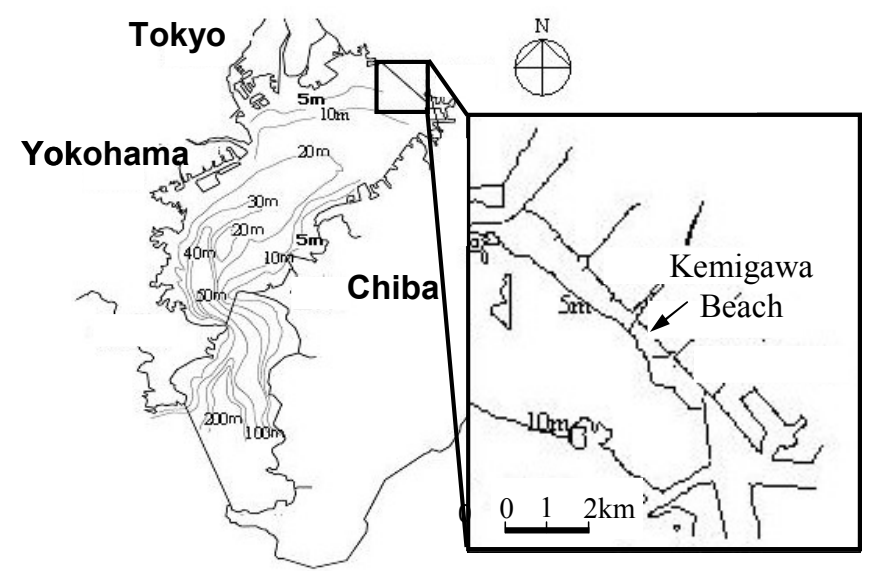

Figure 1. Location of Kemigawa Beach facing Tokyo Bay.

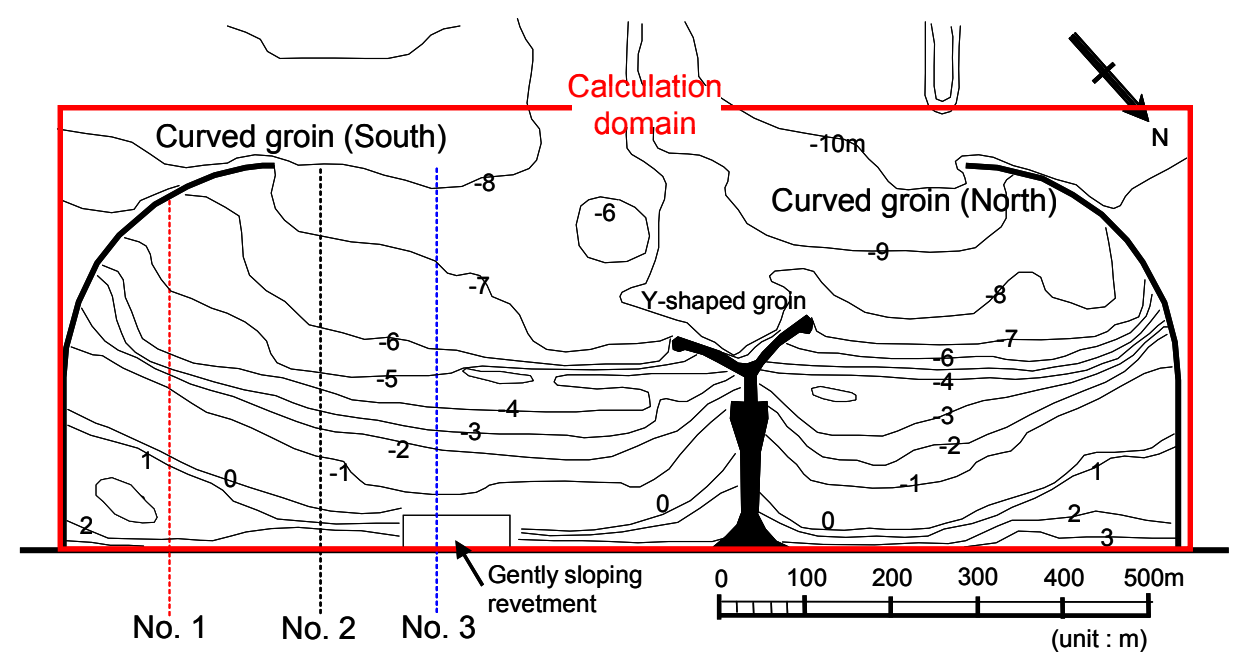

Figure 2. Bathymetry of Kemigawa Beach in 2004.

Figure 3 shows the longitudinal profiles along transect Nos. 1, 2 and 3 shown in Fig. 2, where transect Nos. 1 and 3 are respectively located inside and outside the wave-shelter zone and No. 2 is located in the middle of the beach. Figure 4 shows the corresponding cross-shore distribution of grain size composition along the three transects. 


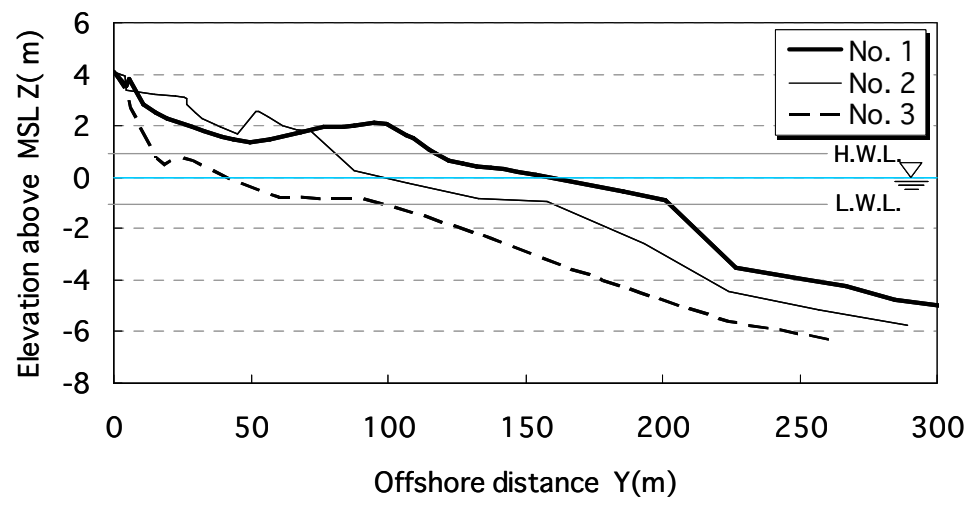

Figure 3. Longitudinal profiles along three transects at Kemigawa Beach.

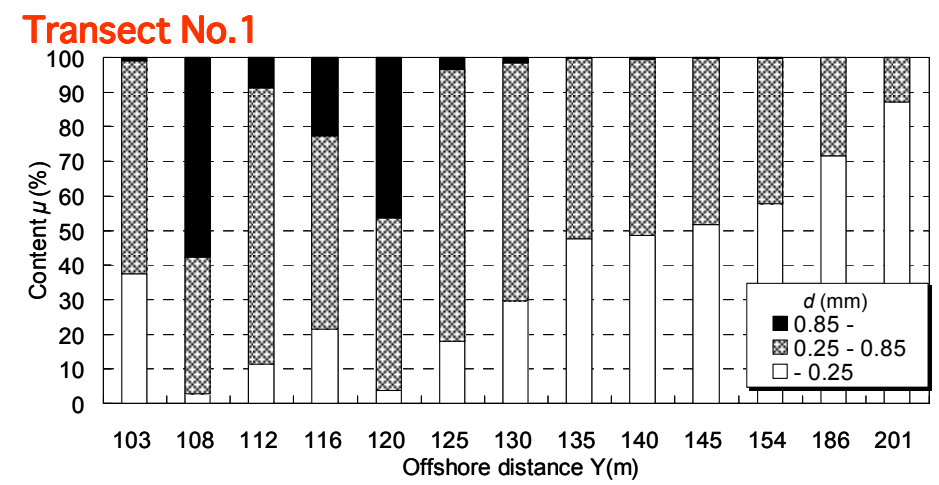

Transect No. 2

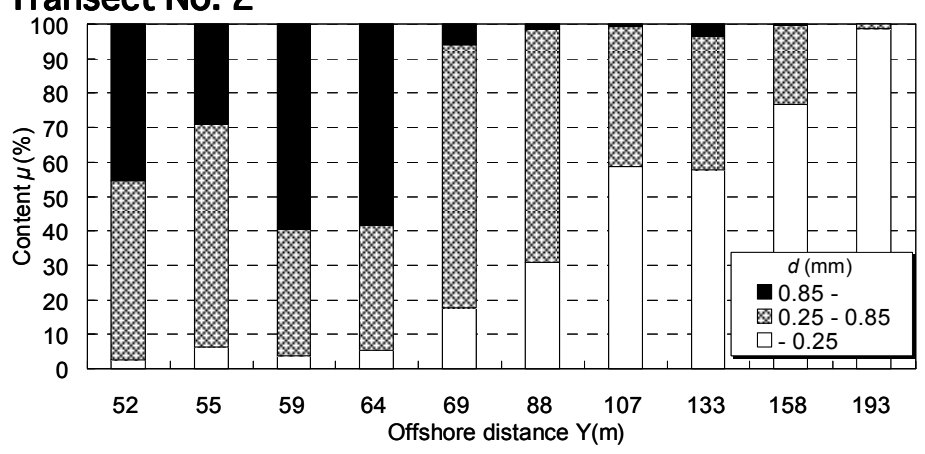

Transect No.3

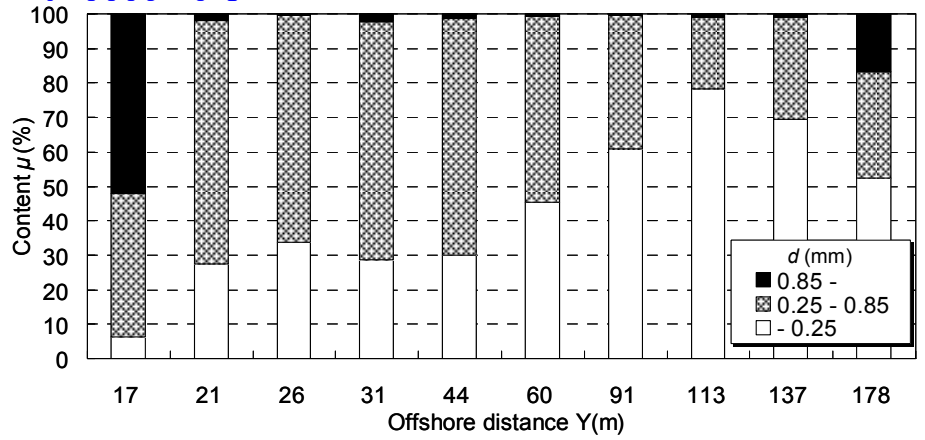

Figure 4. Cross-shore distribution of composition of each grain size along transect Nos. 1, 2 and 3 (measured). 
Along transect No. 1, a foreshore slope of 1/17 above the high water level (HWL) has formed, whereas the seabed slope is as gentle as 1/50 between the HWL and LWL. Because of these differences in slope, coarse sand has been deposited on the shore face, and the offshore seabed with a gentle slope has been covered with fine and medium sand. Similar features can be seen along transect No. 2. Along transect No. 3, located in the erosion zone, coarse sand is concentrated near the shoreline.

Regarding the grain size composition, the relatively steep slope is composed of medium and coarse sand, whereas the gentle slope is mainly composed of fine and medium sand without coarse sand. This is considered to be due to the effect of the longshore and cross-shore sorting of sand by waves; near the downcoast boundary of the curved groins, a large volume of fine sand was deposited, whereas the content of coarse sand increased outside the wave-shelter zone, because fine sand was selectively transported away from the area due to the higher mobility of fine sand than that of coarse sand.

\section{APPLICATION TO KEMIGAWA BEACH}

\section{Calculation conditions}

The calculation domain is a rectangular region $1,300 \mathrm{~m}$ long and $450 \mathrm{~m}$ wide separated by the curved groins. For the calculation conditions, the beach slope in the zone shallower than $-4 \mathrm{~m}$ is assumed to be $1 / 20$, and that in the zone deeper than $-4 \mathrm{~m}$ is $1 / 50$, which are the average measured slopes. The number of grain sizes is assumed to be three, and the typical grain sizes for the grain size ranges corresponding to fine sand and silt $(d \leq 0.25 \mathrm{~mm})$, medium sand $(0.25 \mathrm{~mm} \leq d \leq 0.85 \mathrm{~mm})$ and coarse sand $(d \geq 0.85 \mathrm{~mm})$ are assumed to be $0.106,0.425$ and $0.85 \mathrm{~mm}$, respectively. The contents of the three grain sizes are $35 \%(0.106 \mathrm{~mm}), 50 \%(0.425 \mathrm{~mm})$ and $15 \%(0.85 \mathrm{~mm})$ and the corresponding equilibrium slopes are assumed to be $1 / 60(0.106 \mathrm{~mm}), 1 / 40(0.425 \mathrm{~mm})$ and $1 / 7(0.85 \mathrm{~mm})$. The content of each grain size was determined on the basis of measured values; the sieve analysis of 30 samples taken along transect Nos. 1, 2 and 3 located in the accretion, neutral and erosion zones was carried out to obtain the content of each grain size range. The mean content of the beach material was determined as the average of the contents of grain size along the three transects.

As the initial condition, parallel contours were assumed in the calculation domain, and waves with a breaker height of $H_{b}=1 \mathrm{~m}$, which was determined to be the annual mean wave height, were incident to the initial contours. The equilibrium slope $\tan \beta_{c}^{(k)}$ corresponding to the grain size $d^{(k)}$ was determined from Eq. (11) given $a=0.11$ and $b=0.93$, which were obtained by the least-squares method on the basis of the measured data for Kemigawa Beach. The other calculation conditions are shown in Table 1.

\begin{tabular}{|c|c|c|}
\hline Cases & Kemigawa Beach & Dredging of navigation channel \\
\hline Initial slope & $1 / 50$ & $1 / 20$ \\
\hline Initial grain size and content & $\begin{array}{l}0.106 \mathrm{~mm}, 33 \% \\
0.425 \mathrm{~mm}, 50 \% \\
0.85 \mathrm{~mm}, 15 \%\end{array}$ & $\begin{array}{l}0.125 \mathrm{~mm}, 33 \% \\
0.75 \mathrm{~mm}, 33 \% \\
2.0 \mathrm{~mm}, 34 \%\end{array}$ \\
\hline Equilibrium slope (grain size) & $\begin{array}{l}1 / 60(0.106 \mathrm{~mm}) \\
1 / 40(0.425 \mathrm{~m}) \\
1 / 7(0.85 \mathrm{~mm})\end{array}$ & $\begin{array}{l}1 / 50(0.125 \mathrm{~mm}) \\
1 / 15(0.75 \mathrm{~mm}) \\
1 / 3(2.0 \mathrm{~mm})\end{array}$ \\
\hline Thickness of exchange layer B (m) & 0.054 & 0.150 \\
\hline Breaker height $H_{b}(\mathrm{~m})$ & 1.0 & 3.0 \\
\hline Breaker angle $\alpha$ (deg.) & 0 & 0 \\
\hline Water level & Mean sea level & Mean sea level \\
\hline Depth of closure $h_{c}(\mathrm{~m})$ & 5.0 & 10.0 \\
\hline Berm height $h_{R}(\mathrm{~m})$ & 2.0 & 3.0 \\
\hline Coefficient of sand transport A & 0.3 & 0.3 \\
\hline Coefficient of Ozasa and Brampton (1980) & $1.62 K_{y}^{(k)}$ & $1.62 K_{y}^{(k)}$ \\
\hline $\begin{array}{l}\text { Ratio of coefficient of cross-shore transport } \\
\text { to that of longshore sand transport } \\
K_{x}{ }^{(k)} / K_{y}{ }^{(k)}\end{array}$ & 0.2 & 0.2 \\
\hline Critical slope on land and seabed & $1 / 2$ and $1 / 20$ & $1 / 2$ and $1 / 3$ \\
\hline Calculation domain alongshore (m) & 1,300 & 1,000 \\
\hline Mesh size $\Delta x(\mathrm{~m})$ and $\Delta y(\mathrm{~m})$ & 20 and 20 & 10 and 20 \\
\hline Time step $\Delta t(\mathrm{hr})$ & 0.1 & 0.01 \\
\hline Total number of steps & 20,000 & 60,000 \\
\hline
\end{tabular}




\section{Calculation conditions}

Figure 5 shows the predicted bathymetry in 2004 given the initial parallel contours in 1984 . The advance of offshore contours near the curved groins and the recession of nearshore contours outside the wave-shelter zone with the formation of a scarp are in good agreement with the measured changes. Furthermore, the beach slope near the shoreline became steep and a gentle slope was formed in the offshore zone. Thus, the changes in slope in the cross-shore direction were accurately predicted by the present model along with the longshore changes in the foreshore slope.

Figure 6 shows the predicted longitudinal profiles along the three transects corresponding to the measured profiles in Fig. 3. In the profile along transect No. 3, located in the erosion zone, a concave shape was formed because of the steep slope near the shoreline. In contrast, along transect No. 1, located in the accretion zone, the foreshore slope became steep but a gentle slope was formed in the offshore zone. These features are in good agreement with the measured profiles.

Figure 7 shows the results for the content of each grain size along each transect. It is clarified that the content of fine sand increases and the content of coarse sand decreases toward the wave-shelter zone. Furthermore, the predicted results for the composition of each grain size and the longitudinal slope at each point correspond well to the measured results, as shown in Fig. 4. Thus, the measured results were explained by the present model.

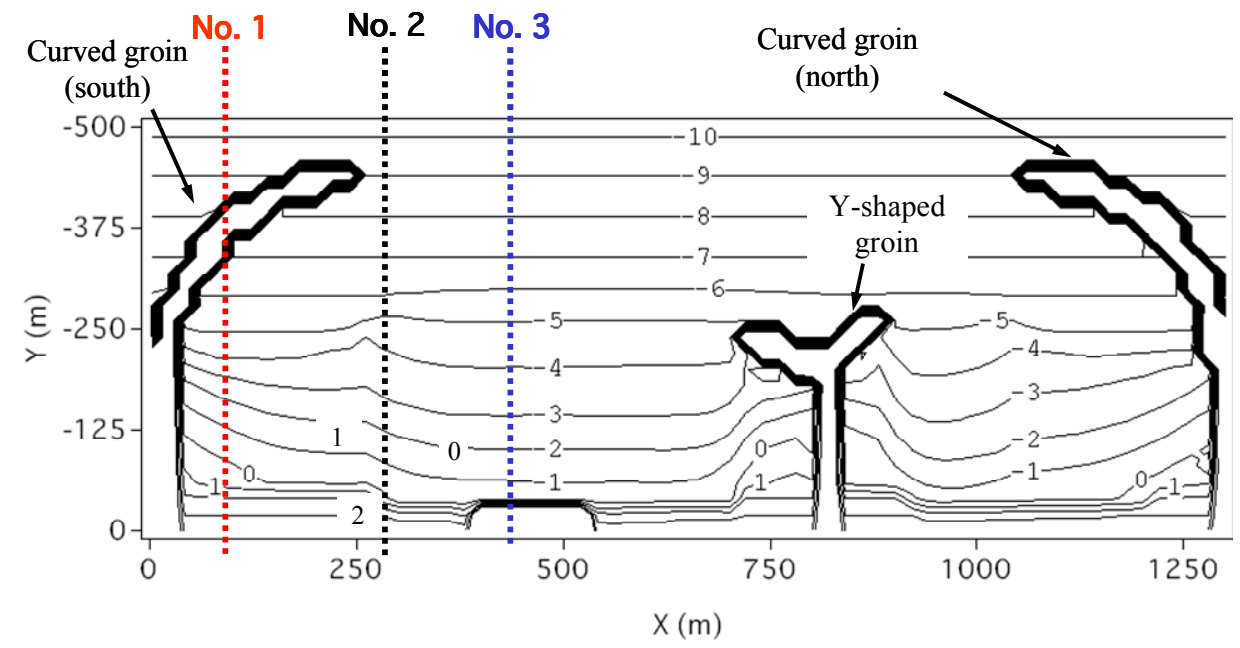

Figure 5. Predicted bathymetry. 


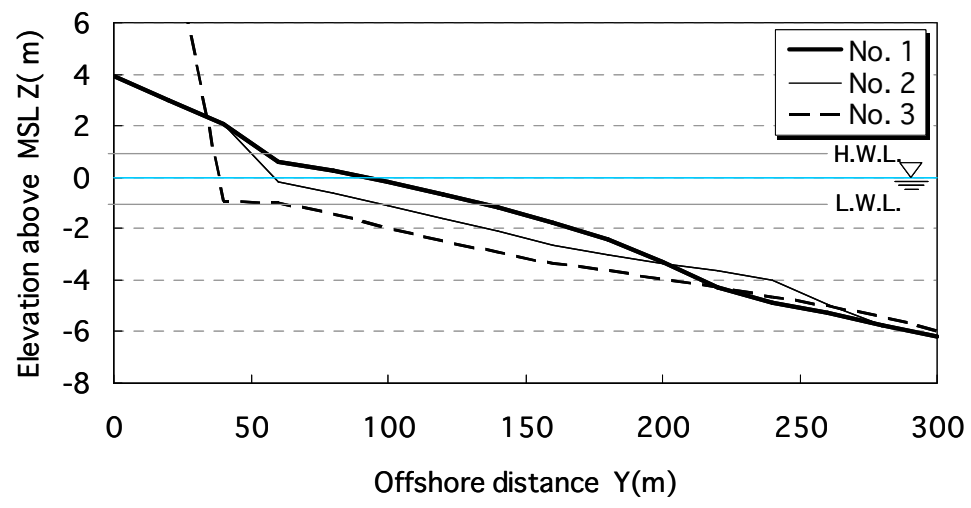

Figure 6. Predicted longitudinal profiles along transect Nos. 1, 2 and 3.

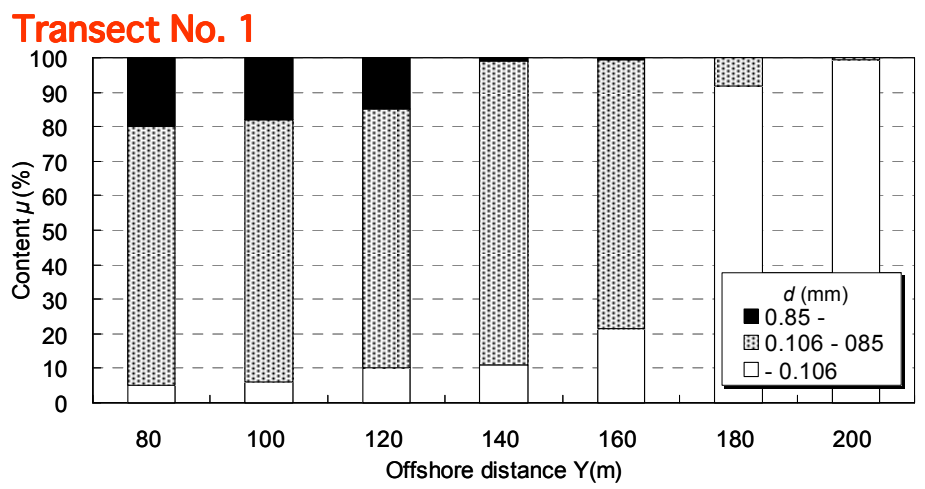

Transect No. 2

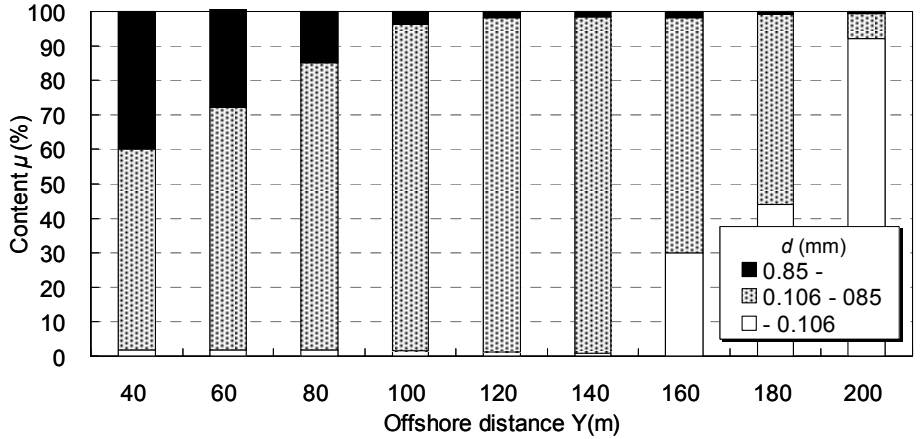

Transect No. 3

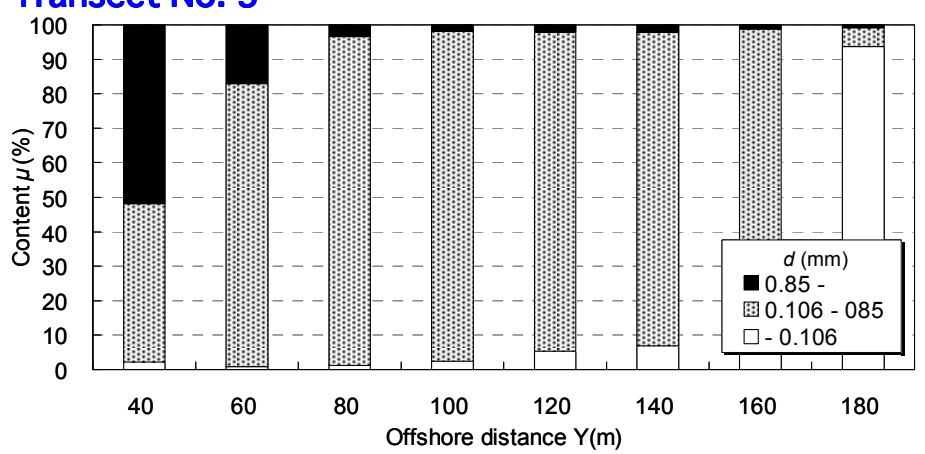

Figure 7. Cross-shore distribution of composition of each grain size along transect Nos. 1, 2 and 3 (predicted). 


\section{PREDICTING DEPOSITION OF FINE SAND INTO NAVIGATION CHANNEL}

\section{Calculation conditions}

Because the present model is advantageous for predicting the transport of sand of each grain size, the selective accumulation of fine sand into a navigation channel was predicted using an imaginary case. In the calculation, three grain sizes are considered, the contents of which are assumed to be $\mu_{1}=33 \%$ $\left(d_{1}=0.106 \mathrm{~mm}\right), \mu_{2}=33 \%\left(d_{2}=0.425 \mathrm{~mm}\right)$ and $\mu_{3}=34 \%\left(d_{3}=2 \mathrm{~mm}\right)$. The calculation domain is a rectangle with $1000 \mathrm{~m}$ length and $500 \mathrm{~m}$ width. The other calculation conditions are shown in Table 1.

\section{Results}

When we assume that waves with breaker height $H_{b}=3.0 \mathrm{~m}$ are incident to the model beach from the direction normal to the initial shoreline, the changes in profile and grain size due to cross-shore sand transport after 20,000 steps are calculated as shown in Fig. 8. Coarse material accumulates near the foreshore forming a berm, whereas the offshore bed is covered with fine material. Furthermore, assuming that the initial bathymetry and grain size distribution before the installation of a breakwater and a groin have longshore uniformity with the cross-shore distribution, as shown in Fig. 8, then they are given by Figs. 9 and 10, respectively.
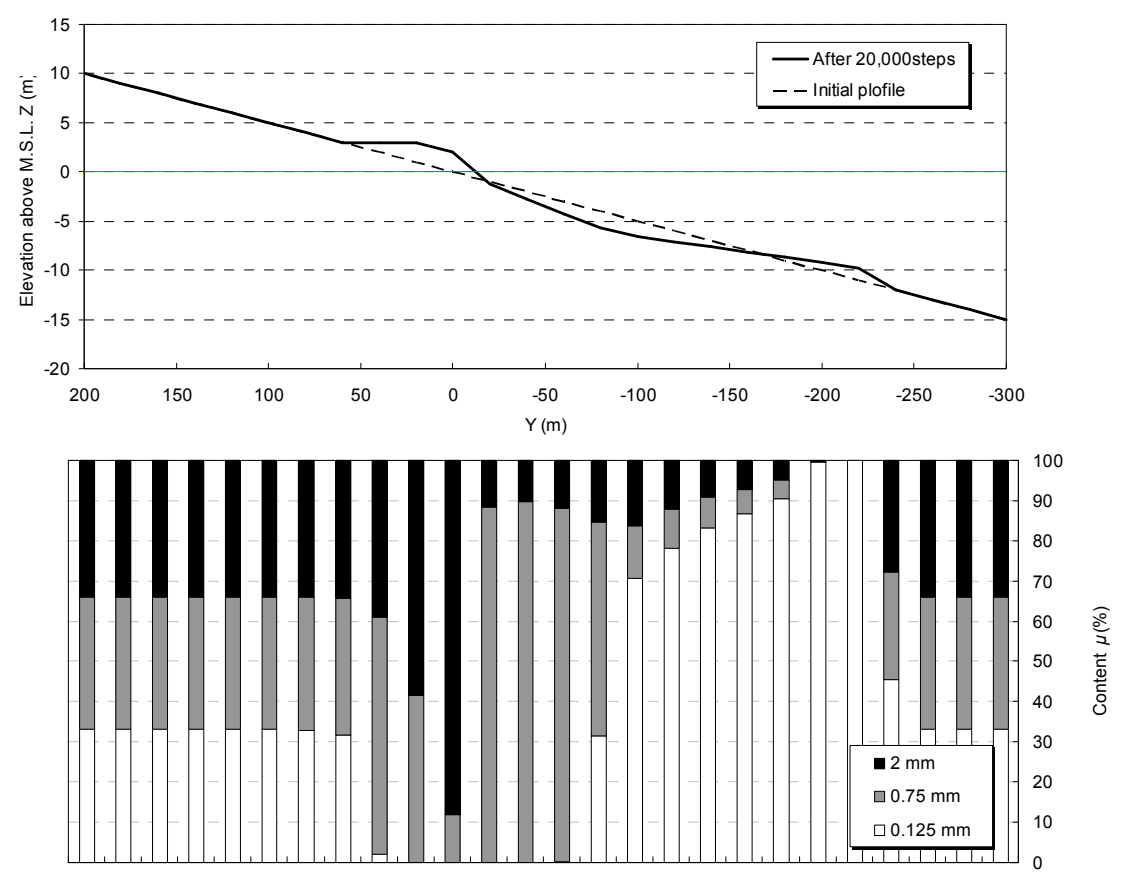

Figure 8. Predicted longitudinal profile and cross-shore distribution of content of each grain size. 


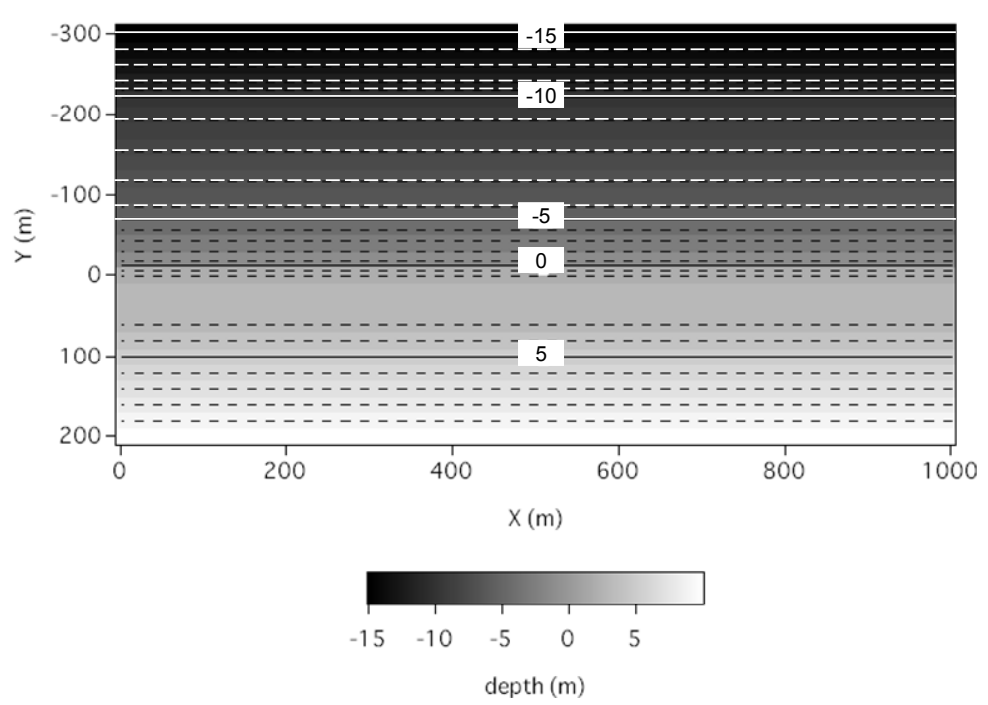

Figure 9. Initial bathymetry before constructing an offshore breakwater and a groin.

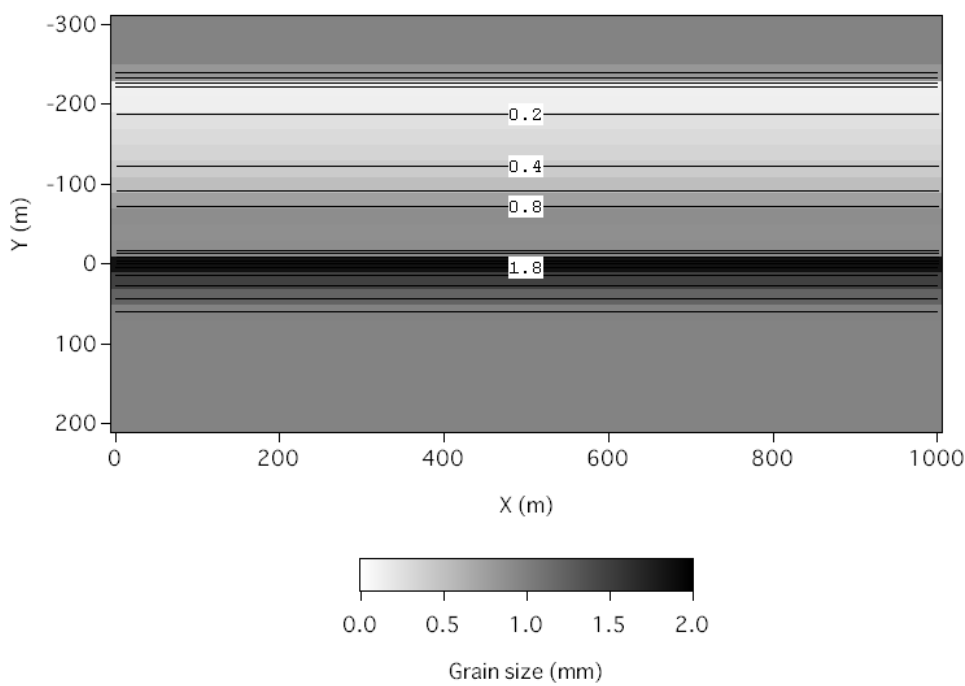

Figure 10. Initial distribution of grain size before constructing an offshore breakwater and a groin. 
Figure 11 shows the predicted bathymetry 20,000 steps after the installation of the offshore breakwater. Sand was transported around the tip of the groin from outside to inside the wave-shelter zone of the offshore breakwater. Immediately to the left of the groin, the shoreline locally receded, and sand was transported further inward toward the port. Figure 12 shows the distribution of the median diameter of grain size corresponding to the bathymetry shown in Fig. 11. Fine sand was selectively transported from outside the wave-shelter zone, far from the breakwater, to near the groin and deposited near the navigation channel offshore of the groin. Erosion occurred immediately to the left of the groin and sand was deposited deep in the wave-shelter zone. Because of the accumulation of sand with coarser grain size near the shoreline, the foreshore slope became steeper than that before the construction of the offshore breakwater.

Figure 13 shows the bathymetry immediately after dredging the sand deposited in the navigation channel. Waves were incident under these conditions, and the bathymetry after 20,000 steps is shown in Fig. 14. Sand was again transported while being deposited in the navigation channel offshore of the groin. The corresponding distribution of the median diameter of grain size is shown in Fig. 15. It was found that fine sand was selectively transported and deposited offshore of the groin whereas the seabed on the nearby coast was covered with coarser material.

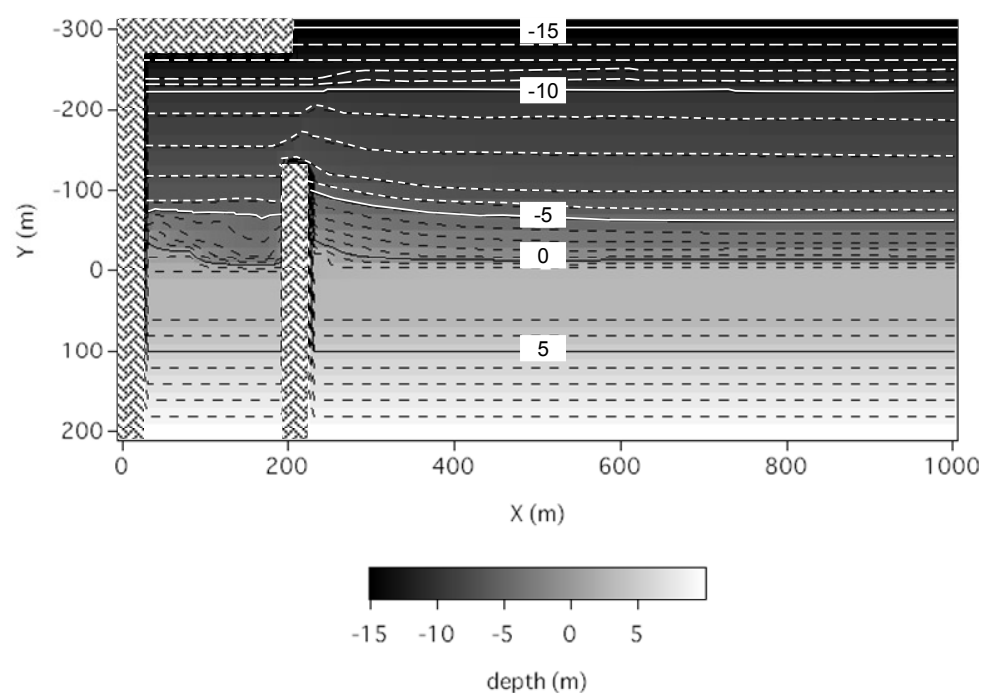

Figure 11. Predicted bathymetry 20,000 steps after the construction of an offshore breakwater and a groin.

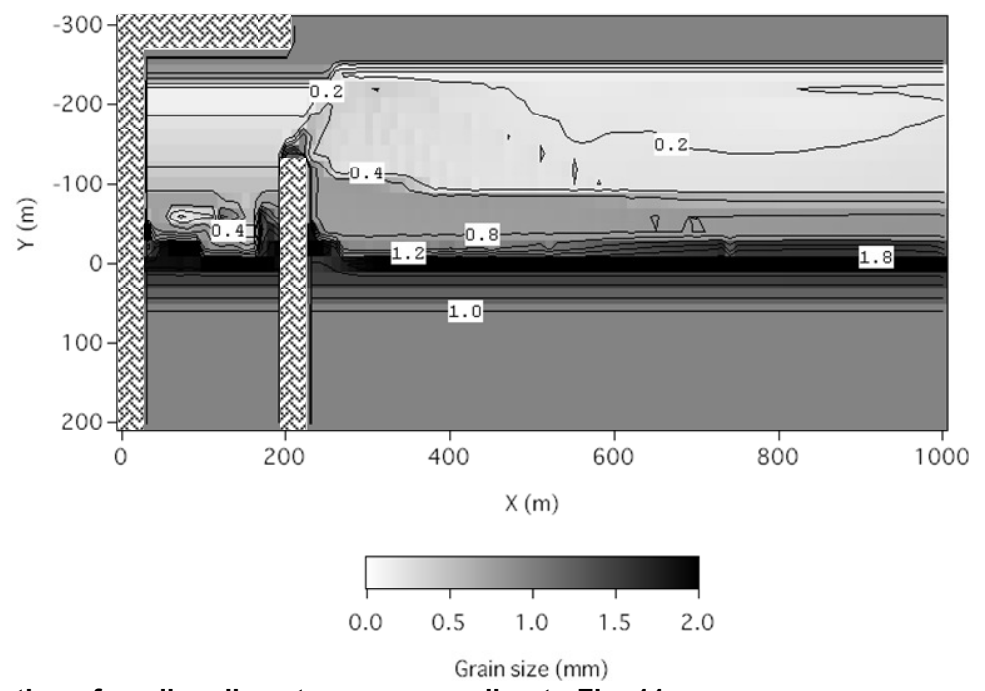

Figure 12. Distribution of median diameter corresponding to Fig. 11. 


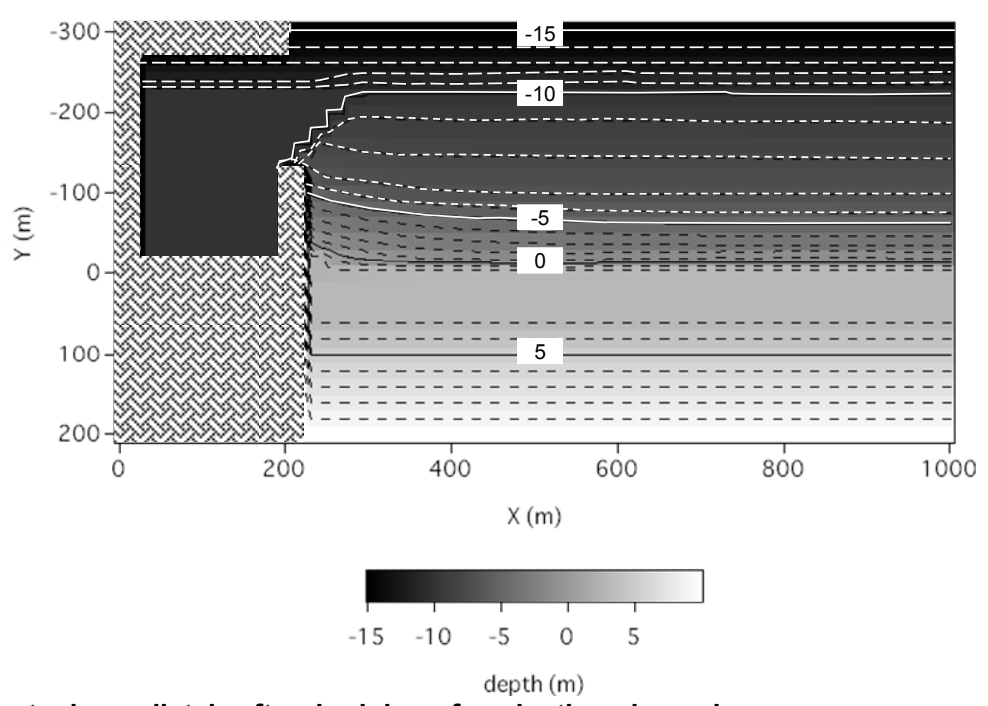

Figure 13. Bathymetry immediately after dredging of navigation channel.

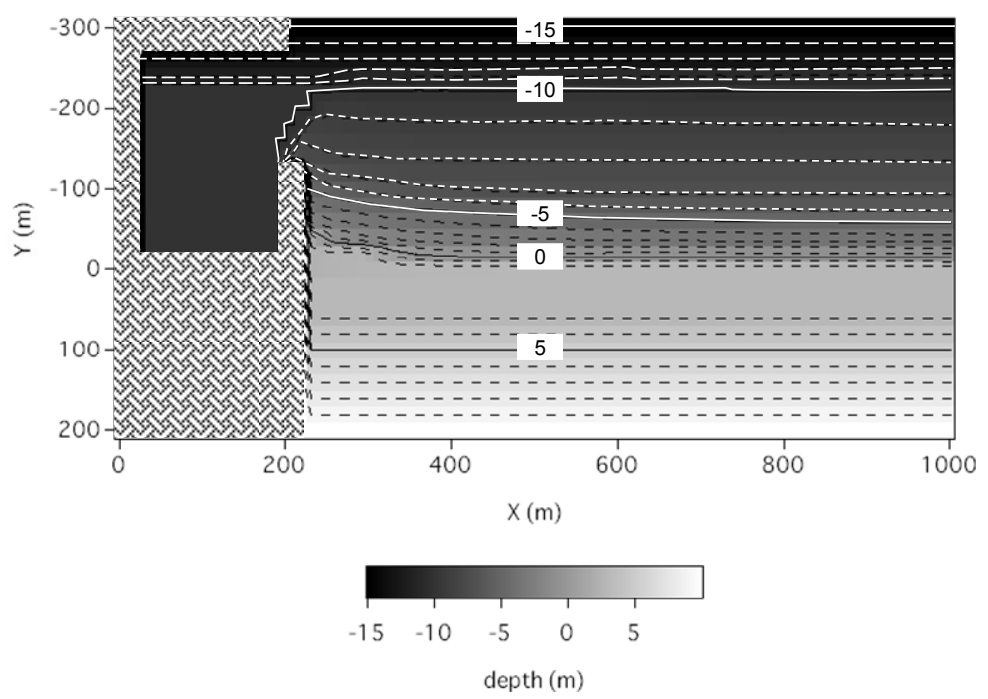

Figure 14. Predicted bathymetry 20,000 steps after dredging.

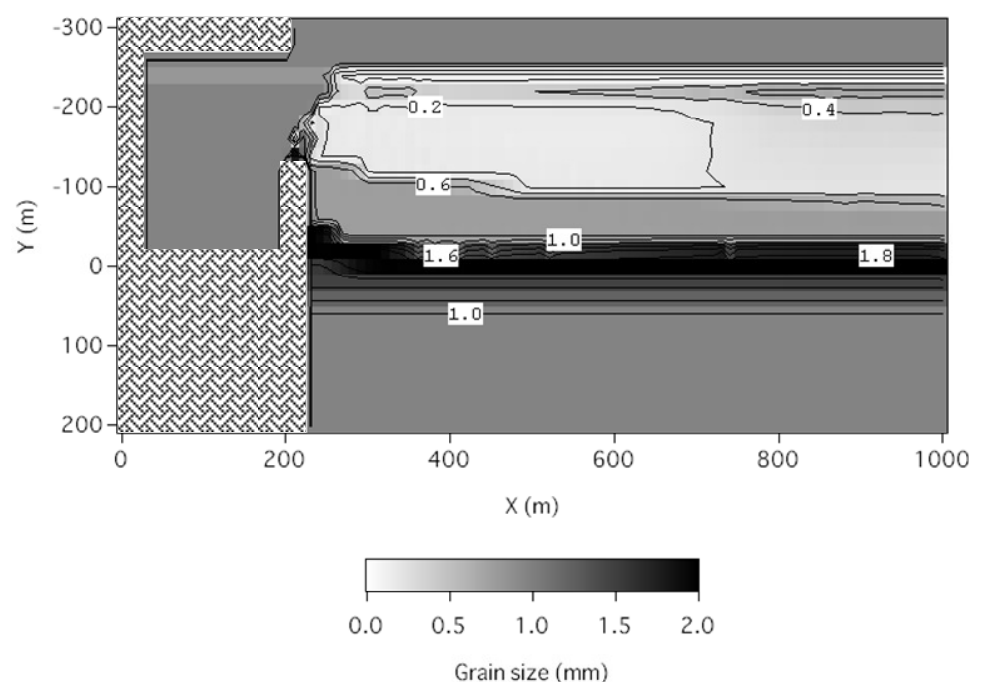

Figure 15. Distribution of median diameter corresponding to Fig. 14. 


\section{CONCLUSIONS}

A model for predicting bathymetric and grain size changes considering the equilibrium slopes corresponding to each grain size and its content was incorporated into the BG model proposed by Serizawa et al. (2007). The model was applied to predict the beach changes at Kemigawa Beach. The cross-shore sorting of sand, in which the grain size gradually decreases with increasing depth, and the formation of a gradually changing longitudinal slope were reproduced. The model was also applied to predict the selective accumulation of fine sand in a navigation channel. It was predicted that fine sand would be selectively transported into the wave-shelter zone, causing the deposition of sand in the navigation channel, whereas the seabed on the nearby coast would be covered with coarser material.

\section{REFERENCES}

Bagnold, R. A. 1963. Mechanics of marine sedimentation, in the Sea, M. N. Hill (ed.), 3, 507-528, Wiley, New York.

Hirano, M. 1971. River-bed degradation with armoring, Proc. JSCE, Vol. 195, 55-56. (in Japanese)

Inman, D. L. and Bagnold, R. A. 1963. Littoral processes, in The Sea, M. N. Hill (ed.), 3, 529-533, Wiley, New York.

Kamphuis, J. W., Davies, M. H., Narim, R. B. and Sayao, O. J. 1986. Calculation of littoral sand transport rate, Coastal Eng., 10, 1-12.

Kumada, T., Uda, T., Serizawa, M. and Noshi, Y. 2006. Model for predicting changes in grain size distribution of bed materials, Proc. $30^{\text {th }}$ ICCE, 3043-3055.

Kumada, T., Uda, T. and Serizawa, M. 2007. Quantitative evaluation of controlling effect of headland on longshore sand transport using model for predicting changes in contour lines and grain size, Coastal Sediments '07, 2446-2459.

Noshi, Y., Kobayashi, A., Uda, T., Kumada, T. and Serizawa, M. 2006. Relationship between local seabed slope and grain size composition of bed materials, Proc. $30^{\text {th }}$ ICCE, 3030-3042.

Noshi, Y., Kobayashi, A. and Uda, T. 2009. Model for predicting bathymetric and grain size changes considering equilibrium beach slopes corresponding to composition of grain size and each grain size, Jour. Coastal Res., 56, 108-112.

Ozasa, H. and Brampton, A. H. 1980. Model for predicting the shoreline evolution of beaches backed by seawalls, Coastal Eng., 4, 47-64.

Sakai, K., Uda, T., Serizawa, M., Kumada, T. and Kanda, Y. 2006. Model for predicting threedimensional sea bottom topography of statically stable beach, Proc. $30^{\text {th }}$ ICCE, 3184-3196.

Serizawa, M., Uda, T., San-nami, T. and Furuike, K. 2006. Three-dimensional model for predicting beach changes based on Bagnold's concept, Proc. $30^{\text {th }}$ ICCE, 3155-3167.

Serizawa, M., Uda, T., San-nami, T., Furuike, K., Ishikawa, T. and Kumada, T. 2007. Model for predicting beach changes on coast with sand of mixed grain size based on Bagnold's concept, Coastal Sediments '07, 314-326.

Uda, T., Yamamoto, Y., Itabashi, N. and Yamaji, K. 1996. Field observation of movement of sand body due to waves and verification of its mechanism by numerical model, Proc. $25^{\text {th }}$ ICCE, 137-150.

Uda, T., Kumada, T. and Serizawa, M. 2004. Predictive model of change in longitudinal profile in beach nourishment using sand of mixed grain size, Proc. $29^{\text {th }}$ ICCE, 3378-3390.

Uda, T., Serizawa, M., San-nami, T. and Ishikawa, T. 2010. Prediction of dynamically stable ebb tidal delta and measures for preventing offshore sand loss, Proc. $32^{\text {st }}$ ICCE. (in press) 